\title{
ENABLING USER PREFERENCES THROUGH DATA EXCHANGE
}

\author{
Steven M. Green * \\ NASA Ames Research Center \\ Moffett Field, California
}

\author{
Dr. Tsuyoshi Goka ${ }^{\dagger}$ \\ Sterling Software Inc. \\ Moffett Field, California
}

\author{
David H. Williams * \\ NASA Langley Research Center \\ Hampton, Virginia
}

\begin{abstract}
$\underline{\text { Abstract }}$
This paper describes the application of data exchange, for integrating user and air traffic management (ATM) systems, to enable user preferences for en-route flights. User preferences may be defined in terms of a fourdimensional (4D) user-preferred trajectory, or a series of profile constraints (e.g., speed, routing, time), depending on user capability. Deviations from the user's preference are often required to meet ATM-system constraints related to capacity, weather, and conflicting preferences of other flights. Progress in reducing these deviations is considered progress toward free flight.
\end{abstract}

This paper describes a process, via user-ATM data exchange, for enabling user preferences in an ATM-based system. An emphasis is placed on determining the minimum deviations necessary to ensure a safe and efficient flow of traffic. Several key system-integration issues are identified and explored in terms of their impact on the data to be exchanged and the roles of the user and ATM systems. Three categories of key data are proposed for exchange, including: user preferences, for one flight or a group of flights; calibration data, to improve trajectory predictions (airborne and ground-based); and ATMsystem state data, to improve the basis for user decision making and preference selection. The paper concludes with a brief description of two experimental evaluations designed to explore data-exchange applications involving current and future systems for flight management and data link.

\section{Introduction}

Projections of strong traffic growth and concerns over the cost of flight operations have led to a strong industry desire to achieve free-flight benefits. ${ }^{1}$ Airspace users (users) want the flexibility to operate their flights

* Research Scientist, Member AIAA.

$\dagger$ Senior Engineer.

$\ddagger$ Research Scientist.

Copyright " 1997 by the American Institute of Aeronautics and Astronautics, Inc. No copyright is asserted in the United States under Title 17, U.S. Code. The U.S. Government has a royalty-free license to exercise all rights under the copyright claimed herein for Governmental purposes. All other rights are reserved by the copyright owner. according to their preferences (e.g., schedule and trajectory), to achieve their specific efficiency needs. However, deviations from user preferences occur as the air traffic management (ATM) system works to maintain separation and manage high-density airspace. These deviations directly impact the economic efficiency of the user's flight operations. The development of a costeffective air transportation system that operates with minimum deviations from user preferences is a fundamental goal of free flight.

In any approach toward free flight, the overall system must address two factors: safety (e.g., separation under instrument flight rules); and an equitable compromise between conflicting preferences of individual flights. The approach must also address the needs of a mixed fleet of aircraft (with a wide variation in capability) since users are reluctant to purchase new or additional equipment without the strong likelihood of a favorable and timely return on their investment capital. One approach to allow users greater flexibility is to transfer separation responsibility from the controller to the pilot. Although technically feasible given the development of new airborne systems and procedures, this approach may be difficult to achieve in high-density airspace and costly to implement for each aircraft. Furthermore, this approach does not address the overall system efficiency issues related to conflicting preferences as flights transit, or arrive at, high-density airspace.

An alternative approach is to change the ATM system to provide a more user-efficient service that works to enable user preferences. ${ }^{2}$ As an unbiased third party, the ATM system could optimize the airspace for all users if it accounted for the preferences of individual users and implemented equitable compromises between flights with conflicting preferences. An improved ATM system offers additional advantages: It lays the foundation for efficient mixed-fleet operations while providing additional flight efficiency benefits for users who invest in greater capabilities such as data link, flight planning, and flight management systems (FMSs). It may also provide a foundation to support greater airborne flexibility in lower density airspace where fewer conflicting preferences would not greatly diminish the overall airspace efficiency for the users.

Substantial progress has been made in developing prototype ATM decision support tools (DSTs), such as 
the Center-TRACON Automation System (CTAS) to improve the efficiency of high-density airspace. ${ }^{3,4}$ These automation tools focus on the traffic management of capacity-constrained airspace as well as the detection and resolution of high-probability conflicts. With knowledge of user capabilities and preferences, these ATM DSTs will be able to assist controllers in adapting to, and taking advantage of, the unique operational characteristics and capabilities of each flight while minimizing deviations due to conflicts between the user-preferred trajectories (UPTs) of two or more flights.

Work is under way at NASA to determine system and procedural requirements necessary to integrate user and ATM systems in order to define, communicate, and enable user preferences for en-route flights with minimum deviations. The approach is to develop prototype automation systems, both airborne and ground-based, and then explore the operational issues through a series of real-time simulations and field tests. An emphasis is placed on the efficient handling of constraints due to high-density airspace involving the ascent, cruise, or descent phases of flight. An "ideal" system with a high level of automation and integration (user and ATM) is assumed as a basis for understanding the maximum utilization of user preferences. From this understanding, simpler approaches for communicating and accommodating user preferences may be evaluated and compared to the ideal system in terms of relative cost and benefit. The lessons learned from prototype evaluations will be used to establish requirements, standards, and recommendations for implementing the most valuable options.

This paper describes the concept in terms of the data to be exchanged, the application of the data, and the roles of user and ATM systems for enabling user preferences. The scope is post-departure with a focus on a dynamic ATM environment with a look-ahead horizon of 20-40 min. The paper begins with a description of a process to enable user preferences in an ATM system, including the userATM interactions to negotiate minimum deviations from the users' preferences. Three key system integration issues are then identified and described. Three categories of data are proposed to support the "negotiation" process: user preferences, calibration data for improved trajectory prediction, and ATM-system state to support the user selection of viable preference options. A brief overview of two proposed experimental evaluations is presented, followed by concluding remarks.

\section{Enabling User Preferences in the ATM System}

The enabling of user preferences will require significant interaction between the users and the ATM system. The users, responsible for the safe and economical operation of their flights, define the preferences for each flight. Preferences may include the UPT for an individual flight as well as the sequence between flights of the same company. Depending on the user's capability, the characterization of a UPT may range from simple profile constraints (e.g., routing, altitude, and speed/time), to a full 4D trajectory. Preference selection may involve the flight crew (pilot), flight planner (dispatcher), or both. The pilot may be aided by cockpit automation (FMS) and the dispatcher may be aided by flight planning automation. User preference selection may take on a variety of forms, depending on the user's needs and the capability of the user's systems. A simple user (e.g., most general aviation operations) may select preferences based on experience and situation, whereas a more sophisticated user (e.g., a major air carrier), may select preferences based on trajectory optimization (best wind route and optimal vertical profile) or airline scheduling considerations. Preferences also include pilot choices for comfort and safety ranging from the "desired" to the "nonnegotiable." In contrast, the ATM system is responsible for the safe, orderly, and expeditious flow of traffic. The ATM system must select and implement the strategies and tactics necessary to ensure separation between flights under instrument flight rules (IFR). These strategies and tactics, developed by controllers at the sector level and traffic management coordinators (TMCs) at the facility level, lead to specific clearances issued to each flight. Controllers and TMCs may also be aided by ATM DSTs that provide advisories for conflict resolution and traffic management.

\section{Steps for Enabling Preferences}

The process of enabling user preferences, within the ATM system, may be divided into nine steps representing key user and ATM-system actions. The user first (1) selects preferences and then (2) communicates them to the ATM system. Third (3), the ATM system predicts and updates trajectories for all flights based on the user's preferences and ATM-system state. Fourth (4), the ATM system analyzes the latest trajectory predictions to determine delays and probe for conflicts. Fifth (5), the ATM system forms a delay strategy, or conflict resolution, as needed. Sixth (6), the ATM system issues clearances to affected flights. Seventh (7), the affected flights execute the clearance instructions (or negotiate for safety concerns). Eighth (8), the ATM system monitors conformance and (9) updates users on airspace status (e.g., delays). The process then repeats itself as users update their preferences (due to changes in their mission or ATM constraints) or as the ATM system changes its state (due to changes in airspace, weather, or user preferences).

These steps point to the beneficial applications of key technologies. Ground-based flight planning tools and the FMS can assist the user in determining preferences (step 1) while the FMS may also improve the accuracy with which aircraft conform to clearances (step 7). Data link will provide the infrastructure for timely and low workload data exchange (steps 2, 6, and 9) between the cockpit and the ATM system (air-ground) and between 
the flight planner and ATM system (ground-ground). Finally, ATM DSTs fill a key role in assisting controllers and TMCs in making good use of the preference data and capabilities of individual users (steps 3, 4, 5, and 8).

\section{Trajectory Negotiation}

Ideally, users prefer to operate without ATM constraints, as the default state, with constraints added only when necessary. To the extent that this "ideal" world is possible, figure 1 depicts the dynamic user/ATM-system interaction required to determine the minimum constraints on the arrival time and trajectory of a flight. This process is the basis for the experimental evaluations described in a later section. The process begins, for each flight, with the initialization of preference and calibration data prior to departure. Calibration data are used to improve the trajectory prediction accuracy of both user and ATM DSTs. Once a flight is active (departed), the aircraft state and calibration data are updated as needed to maintain the accuracy of trajectory predictions. The latter (main) stages are then invoked (or repeated) as needed to adapt trajectories to changes in the ATM system and user preferences. The process ends with the flight's arrival at the destination.

The main stages are grouped into two negotiation processes, one for scheduling and the other for profiles. ${ }^{5}$ The scheduling process detects and resolves highprobability conflicts in preferred arrival times while the profile process detects and resolves high-probability conflicts (loss of legal separation) along the preferred trajectories. These negotiation processes are an extension of the traffic management and controller decision-making processes that take place today. The term negotiation is used to emphasize the concept of basing the ATM system analysis (of delays and conflicts) on trajectory predictions that reflect user preferences. The negotiation processes generate constraints only when needed; otherwise the user's preference is honored. Although the negotiation process may determine that a UPT is not in conflict, some cases may require a certain level of clearance conformance to avoid deviations that may lead to other conflicts.

The order of negotiation is time (first), if needed, then trajectory (second). There is little benefit to flying an optimum best-wind route trajectory into a "known" arrival delay. Scheduling applies only to situations requiring traffic management [e.g., arrival metering or miles-in-trail (MIT)], which are constraints typically associated with the transition to high-density terminal airspace. The scheduling process benefits the users by providing an equitable and efficient distribution of the delay when significant delays are predicted with high probability. ${ }^{6,7}$

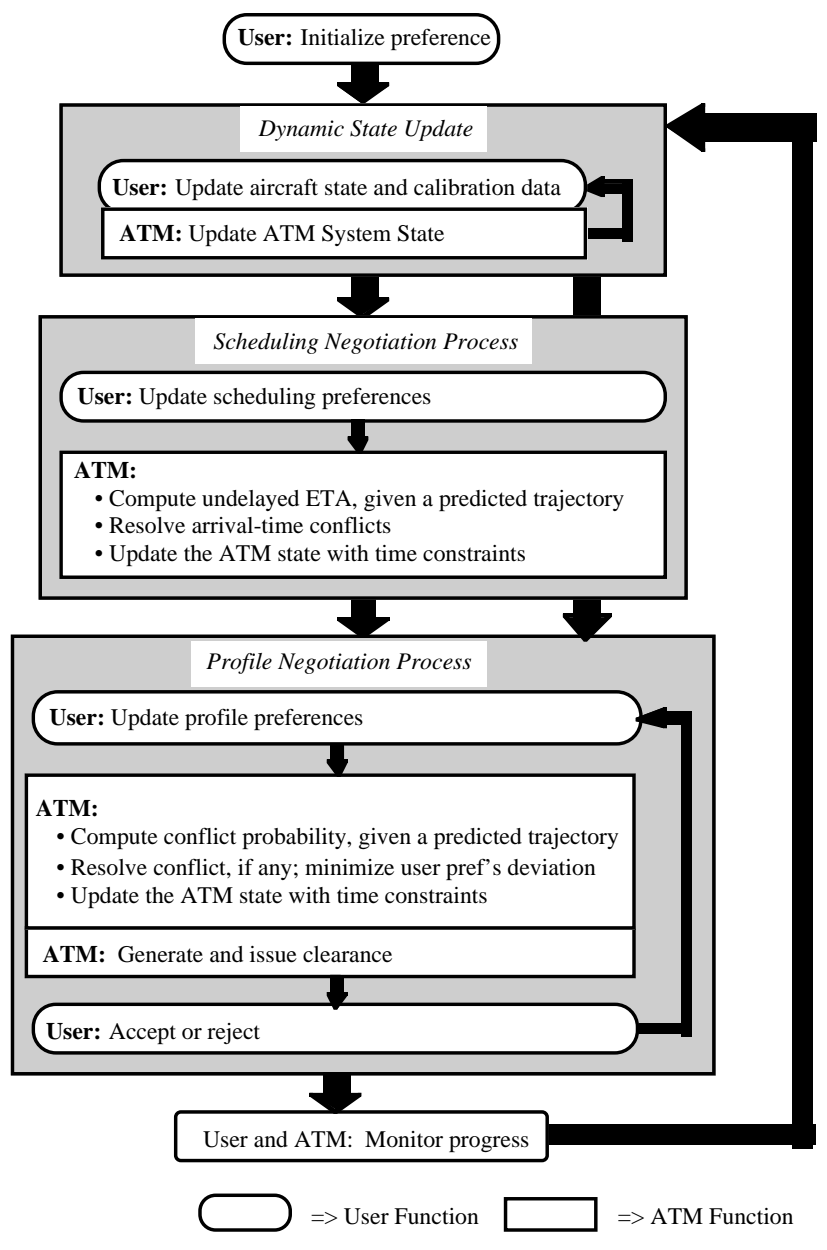

Figure 1. Functional Flow Chart of Proposed Data Exchange Concept

The scheduling negotiation begins with an update of user preferences. The ATM system then determines the traffic demand by updating its prediction of the "undelayed" trajectories for each flight at a reference waypoint. This reference point may range from a runway/approach fix, for the ultimate in free-flight flexibility, to an upstream metering fix, for a more near-term application. The ATM system compares its update of the traffic demand with its model of the airspace capacity to determine delay. Highprobability delays are then distributed among individual flights by issuance of a scheduled time of arrival (STA). If a flight's preference does not result in time conflicts, then no constraint is necessary. The scheduling process repeats continuously to reflect updates in user preferences and ATM status. ATM DSTs such as the CTAS Traffic Management Advisor (TMA) and Descent Advisor (DA) can assist Traffic Management Coordinators (TMCs) and controllers in setting, and meeting STAs for aircraft with and without FMS. 8,9

The trajectory negotiation process also runs continuously, in parallel with the scheduling process, while using any 
STA constraints as input. The trajectory process is similar to the scheduling process with two main differences. The former must analyze user preferences with some flights under the influence of an STA constraint, whereas the latter analyzes undelayed preferences. Second, the primary focus of the trajectory process is to detect and resolve high-probability conflicts (loss of separation along a trajectory) whereas the scheduling process focuses on conflicts in arrival time due to capacity limitations.

The trajectory negotiation process begins with another opportunity for users to update their preferences and optimize for any STA constraints. The ATM system must then update its trajectory predictions to represent any STA constraints (the airspace may contain a mix of STAconstrained arrivals and undelayed overflights). The ATM system must then analyze the trajectories to detect and resolve any high-probability conflicts with minimum deviations from the user preferences. ${ }^{10,11}$ The process results in a set of $4 \mathrm{D}$ trajectory constraints, to be issued as clearances, for only those flights that are in conflict.

The effective time horizon for this process varies with aircraft pair, based on conflict geometry and trajectory prediction accuracy. The trajectory accuracy will vary with aircraft type (capability), phase of flight, atmospheric prediction accuracy, and the accuracy of aircraft state estimation. ${ }^{12}$ In addition, the trajectory prediction accuracy also varies with the accuracy of "trajectory intent" knowledge which is why integration with the scheduling process is critical for accurate conflict prediction. Early conflict resolution for high-probability conflicts will result in more efficient conflict-free trajectories. ATM DSTs such as DA have the potential to assist controllers in accurately meeting STAs while resolving conflicts, with minimum interruptions to the planned trajectory caused by inadequate knowledge of trajectory intent. ${ }^{9}$

\section{$\underline{\text { System Issues }}$}

Consideration of these interactions between the user and the ATM system raises several interesting systemintegration issues, including the coupling between preference selection and ATM-system state, the accuracy of trajectory predictions, and the roles for negotiation.

\section{Coupling of User Preferences and ATM-System State}

The coupling between preference selection and ATMsystem state is analogous to the "the chicken and the egg." The effectiveness of user-ATM negotiation improves as the participants gain a better understanding of their counterparts' positions. Users need to have a reasonably accurate picture of the ATM-system state, in terms of how ATM-system constraints may affect their flights, in order to select effective preferences. For example, knowledge of the predicted arrival delays at a destination could influence a user's choice of routing into the terminal area. Depending on the effective time horizon of the delay predictions, the information may influence the user's choice of routing prior to departure or in flight. In addition to the coupling to user preferences, ATM-system state information also provides the users additional benefits in terms of user resource planning (e.g., ground support services). From the ATM-system point of view, accurate knowledge of a flight's intent is critical to the prediction of delays and conflicts. Given this coupling (between ATM-system state and user preferences) and the dynamic nature of the ATM system, an iterative approach is needed to allow both the ATM system and the users to update their contributions to the system planning. This iterative approach can be achieved by a system that facilitates the real-time update of user preferences and ATM-system state.

\section{Trajectory Prediction Accuracy}

The second system issue concerns trajectory prediction accuracy and the similarity between user and ATM predictions. The prediction accuracy is critical for efficient resolution of conflicts and the fair distribution of delay when traffic demand exceeds airspace capacity. Regarding conflicts, greater trajectory prediction accuracy provides two benefits. First, fewer resolutions (trajectory deviations) will be needed because the ATM DSTs will be able to distinguish the "high" vs. "low" probability conflicts. Second, the greater accuracy will lead to increased confidence in maintaining separation and with it, the potential for more efficient resolution techniques and a reduction in the "buffer" controllers use to protect against uncertainty. Greater similarity between ATMsystem and user predictions will provide two benefits. First, it will help minimize any differences in traffic demand expectations between the user and ATM system. Second, it will ensure that the ATM prediction is consistent with the trajectory that an FMS-equipped aircraft would fly. Both accuracy and similarity can be achieved through data exchange by combining the most accurate sources of data from the users and the ATM system (e.g., winds, aircraft state, and preferences/intent) and ensuring that both the users and the ATM system use the same data.

\section{ATM and User Roles for Negotiation}

The third system issue concerns ATM and user roles for negotiation. As mentioned earlier, the characterization of a user's preference will vary with the user's trajectoryplanning and communications capabilities. A fundamental role for the ATM system is to accommodate the unique preferences for a user fleet of mixed capabilities. This accommodation can be achieved by the concept of "agency."

Under the agency concept, ATM DSTs serve as an unbiased agent for all users. The DST provides the foundation to translate the various UPT characterizations 
into a common standard for ATM-system analysis. This common standard is the trajectory prediction process. If done correctly, the ATM-generated trajectories would be close to the UPTs.

Within the agency concept, the ATM DSTs maintain the user preference data "on file" and distribute the data to the appropriate sectors and ATM facilities. In essence, the preference data follows a flight throughout the ATM system like a flight plan. The difference is that the preference data are stored in parallel to the classic flight plan data, and are easily updated by the appropriate user representatives (pilot and/or flight planner) via air-ground or ground-ground data link. The preferences may be defined in terms of preplanned settings (as a function of operator, aircraft type, and flight), flight plan settings, and/or real-time updates from the pilot or flight planner. This approach provides the ATM DSTs with access to the latest user-preference data while allowing the user an easy way to update preference data. If user-preference data are not available, then the ATM DST defaults to its own best estimate. As an example, CTAS employs a default database of speed profile preferences as a function of aircraft type and operator.

A related user issue concerns the pros and cons of characterizing UPTs in terms of full 4D trajectories as opposed to profile constraints. The primary advantage of user-supplied trajectories is that they leave no question as to the intent of the user. Several disadvantages, however, are evident from an ATM-integration point of view.

First, if the ATM system is to be responsible for separation, the user-supplied trajectories must be synchronized with the ATM system's trajectory prediction process. A difference of 5 to $10 \mathrm{sec}$ is equivalent to about 1 n.mi. of position uncertainty (or 20 percent of the current minimum en-route separation criteria).

Second, user-supplied trajectories must be updated, as needed, to reflect changes in the state of the aircraft, atmosphere, and ATM system (e.g., STA). This is particularly challenging in highly dynamic transition airspace where deviations occur often. To accommodate preference updates (as currently implemented in CTAS TMA scheduling ${ }^{13}$ ), the user must be capable of supporting at least three trajectory options in parallel (fig. 2 ). One trajectory must reflect the current clearance for pilot guidance, the second must reflect an undelayed UPT, and the third must reflect an STA-constrained UPT. If the user wants to negotiate the arrival schedule, the undelayed UPT must also account for the variation of ETA across runway options.

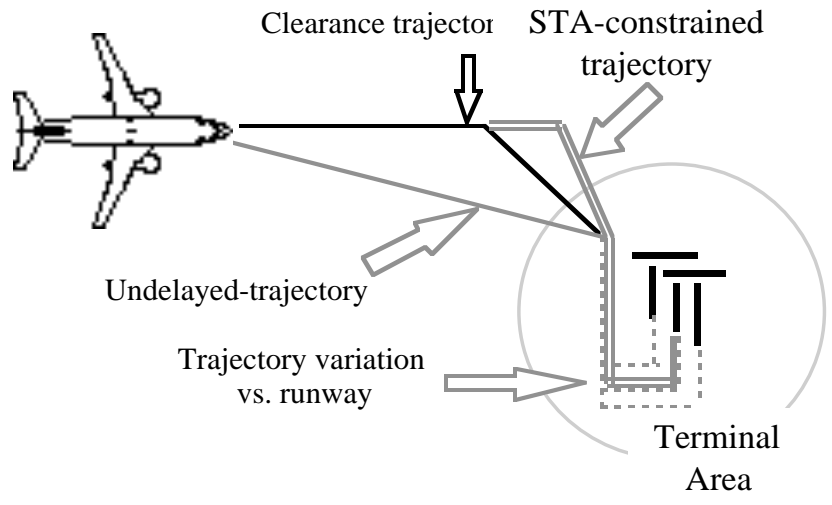

Figure 2. CTAS trajectory updates in transition airspace.

Third, user-supplied trajectories must account for any nonnegotiable ATM constraints (such as STA, crossing altitude, and routing). The air-ground communication required to synchronize UPTs with dynamic ATM constraints could be cumbersome and time consuming. The update process would pose a data link-system performance challenge to guarantee timely updates of ATM-constrained UPTs. If the UPT happens to be inconsistent with a nonnegotiable ATM constraint, then the UPT cannot be used directly for ATM-system analysis (of delays or conflicts). In that case, the ATM system would have to decompose the UPT, salvage any viable profile characteristics, and then generate its own version of the UPT.

One advantage of profile constraints over full 4D trajectories is that constraints allow the ATM system the flexibility to quickly adapt the user's preferences to dynamic changes in ATM state. Even if some users were capable of providing the ATM system with timely updates of valid, time-synchronized UPTs, the ATM DST must still maintain the capability of generating UPTs (under the concept of agency) to provide service for the remaining users. It is possible that the user-supplied trajectory approach may not provide enough user benefit over the profile constraint approach to economically justify the added complexity required of the supporting systems (FMS, ATM DST, and data link).

\section{Data to Be Exchanged}

This section proposes a set of data to be exchanged between the user (pilot and/or flight planner) and the ATM system. The data are summarized in three tables based on application: user preferences (Table 1), trajectory calibration (Table 2), and ATM-system state (Table 3). The data sets and applications were defined based on experience gained in the design and evaluation of CTAS, including a series of real-time-simulation and field-test experiments involving integration with FMS, data link, and user flight planning. Some of the applications have already been implemented within CTAS 
and tested in simulation and in the field. ${ }^{5,9,12}$ The data set is not proposed as a fixed product for all users, but rather as a menu of ATM-system inputs and outputs that may have a direct effect on preference and clearance decision making. Users may select the data that best represents their needs and fits within their capabilities. Most of the contents of this section may be implemented, with slight modifications, under current data-link applications for Automatic Dependent Surveillance and Controller Pilot Data-Link Communication. ${ }^{14,15}$ The remaining messages, involving exchange between the ATM system and user flight planners, would be incorporated within groundground applications. ${ }^{16}$

\section{$\underline{\text { User Preference Data }}$}

User preferences (for the dynamic environment) are listed in Table 1, followed by a discussion of each item.

Table 1. User Preferences

\begin{tabular}{lc}
\hline \hline \multicolumn{1}{c}{ Data } & $\begin{array}{c}\text { Communication } \\
\text { Direction }\end{array}$ \\
\hline Desired Time of Arrival (DTA) & User $>$ ATM System \\
Desired Runway & User $>$ ATM System \\
Runway Delay Weighting & User $->$ ATM System \\
Desired Sequence & User $>$ ATM System \\
Flight Priority & User $>$ ATM System \\
Arrival Delay Weighting & User $>$ ATM System \\
Flight Constraints & User $>$ ATM System \\
Preferred Trajectory & User $>$ ATM System \\
Trajectory Deviation Weights & User $>$ ATM System \\
\hline \hline
\end{tabular}

Desired Time of Arrival (DTA). DTA represents the user's desired undelayed arrival time. Within CTAS, this time is used to represent a flight's demand for an airspace resource (e.g., runway) for determining sequence. As a flight approaches its destination, its DTA may vary with changes that occur in the desired speed profile, desired routing, aircraft/clearance state, and atmospheric state. If a user's priorities are to maintain a target time of arrival (e.g., for user scheduling considerations), the user may update the preferences (such as speed profile) accordingly. DTA is updated continuously, based on the actual progress of the flight. If a target time is not achievable given the available options in speed and routing, then the DTA simply reflects the time that is closest to the user's target yet operationally feasible.

Although it may not be practical for some users to provide ATM with DTA, because of the computational and data communication requirements, it is a routine computation for CTAS automation. One form of agency is for the ATM automation to perform DTA computations based on user input, including cost index (or preferred speed profiles) and routing. These alternative user preferences would lead to ATM computational results that are close to what the user would have computed for DTA if so equipped. Field testing of the CTAS DA has demonstrated the feasibility of this approach with measured meter-fix arrival time prediction accuracy on the order of 15 seconds. ${ }^{9}$

Desired Runway. Desired runway data define the user's runway preferences as a function of airport arrival configuration. The desired runway is a useful preference for minimizing taxi time. The actual runways in use at the destination airport may vary over the course of a flight. Depending on the runway configuration, the user's preferred runway may be different.

Runway Delay Weighting. Runway delay weighting data allow users to specify their preferred runway in terms of expected delay per runway. This indicates the maximum delay a user is willing to accept before changing the runway preference. These data allow the user to define the tradeoff between taxi time and in-flight delay.

Flight Sequence. Flight sequence data allow a user to specify the preferred order of arrival within the user's own arrival bank. This preference is useful for maximizing bank integrity and minimizing bank time (i.e., exchange of passengers/cargo and aircraft servicing).

Flight Priority. Flight priority data allow the user to specify a situation that requires special handling of the flight. Included would be information such as emergencies (to allow undelayed, priority handling) and maximum delay acceptable before deviation to alternate airports .

Flight Constraints. Flight constraint data allow the airline to specify operational constraints (e.g., speed, altitude, routing, runway) that limit the performance envelope or trajectory options for a flight. These limitations are used in the calculation of preferred traject ories.

Preferred Trajectory. Preferred trajectory data allow users to specify their preferred trajectory (UPT) to meet ATM constraints. The ATM system must be able to analyze the traffic situation based on the user's preference input. The most general form of a UPT description is a 4D trajectory. These trajectories may be generated by airborne (FMS) or ground-based flight-planning automation. Alternatively, UPTs may be computed by ATM based on the user's preferences (routing, altitude, and speed). ATM-generated UPTs must consider several factors to accurately model the speed and altitude profiles.

User-preferred speed profiles [e.g., Mach/indicated airspeed (IAS) for jets], vary over the flight. In general, 
speed preferences may be divided into climb, cruise, and descent speed profiles. These speeds are usually chosen based on considerations of time, fuel, and operational procedures. Many users operating FMS-equipped types employ a cost index (defined as a ratio of fuel cost to time cost) to determine speed profiles. The relationship between speed profile and cost index, usually determined by the airframe manufacturer, may vary with factors such as weight, altitude, wind, and temperature. As a result, desired cruise speed will vary over long distances. User speed preferences could either be defined directly, for each phase of flight, or indirectly through cost index. The cost-index approach would require the users (or airframe manufacturers) to provide the ATM system with the relationships necessary to decode cost index into speed profiles. Once these relationships are defined up front for each aircraft type, this one parameter could provide the key to the desired speed profile of an entire flight.

If the ATM system is to generate UPTs based on speed preferences, altitude profiles must be adequately modeled to minimize errors in calculating true airspeed. Altitude profiles may vary in terms of type and magnitude. Profile types may be defined by power settings, flightpath angles, or ascent/descent rate. The magnitude refers to the controlling value (e.g., climb power, three degrees, or 1000 feet per min). Profile modeling may vary significantly across aircraft types. For example, CTAS testing has revealed the following descent-profile preferences.

Jet operators generally prefer to descend at near-idle power, with the descent profiles varying with speed and wind. Small power variations are used for de-icing, when necessary, and for smoothing the transition into and out of the descent. Some descent segments are flown with power to achieve a target descent rate or angle.

Prop operators generally prefer to descend with power and use simple flightpath ratios (e.g., 4 n.mi. per 1000 feet) independent of speed and wind. Shallow profiles allow pilots to fly predictable and controllable descents under a variety of winds and speeds. Prop aircraft, particularly turboprop commuters, are flown with poweron during descent to increase the descent range, enabling the aircraft to maintain a higher speed in descent for a longer period of time. Piston-prop aircraft are flown in a similar manner to prevent excessive shock cooling of high-performance engines. Turboprop-commuter operators typically fly a maximum airspeed "barber-pole" profile. Although most prop aircraft do not have Mach meters, the airspeed indicator adjusts the barber-pole to indicate the airspeed limit due to compressibility or dynamic pressure, whichever is less. By flying a barberpole/IAS profile, prop pilots fly a "pseudo" Mach/IAS speed profile. The combination of shallow descent and barber-pole speed decreases the block time of these types by several minutes while increasing passenger comfort with smaller descent rates.

Trajectory Deviation Weightings. Trajectory deviation weightings data allow users to indicate the cost penalty associated with a representative set of deviations from the UPT. By providing this data up front to the ATM automation, the user's preferences are represented over a large range of tactical options while allowing the ATM system to resolve problems quickly without the communication overhead associated with real-time negotiation.

\section{$\underline{\text { Calibration Data }}$}

A high level of modeling precision between the user and ATM system, and a high level of accuracy between the user/ATM system and the actual environment are desired. Calibration may be accomplished in two ways. The approach presented herein is to exchange actual model data (atmospheric characteristics, aircraft performance, and pilot procedures). An alternative approach is to exchange a 4D trajectory supplemented by air-mass and inertial velocities, the combination of which would enable the receiving automation to infer key performance and atmospheric data.

Table 2. Calibration Data

\begin{tabular}{ll}
\hline \hline \multicolumn{1}{c}{ Data } & \multicolumn{1}{c}{ Communication } \\
& Direction \\
\hline Aircraft State & User $->$ ATM System \\
Aircraft Performance & User $->$ ATM System \\
Arrival Performance & User $->$ ATM System \\
Pilot Procedures & User $->$ ATM System \\
Atmospheric Characteristics & User <-> ATM System \\
\hline \hline
\end{tabular}

Aircraft State. Aircraft state data include position, velocity (airmass and inertial), and weight of the airplane. These data are used to improve the accuracy of the initial conditions for trajectory prediction. The velocity data are particularly important during maneuvers (turns, ascent/descent, and accelerations) when large errors occur in radar-track based velocity estimates. ${ }^{12}$ Although the radar-track-based position estimate is generally within a mile, the velocity vector estimate may be in error by as much as 30 degrees and/or 100 knots, or more, during maneuvers. The velocity data may also be used for monitoring the progress and clearance conformance of a flight. Additional intent data, from FMS-equipped aircraft, could also be used to update the ATM system during clearance deviations such as storm-cell avoidance. The weight data are used to improve the ascent and descent profile predictions. Although velocity updates are needed in real time, the weight data are not time critical. A relatively high accuracy could be achieved with pre- 
flight estimates of takeoff gross weight and fuel-burn (or predicted landing weight and time of flight).

Aircraft Performance. Aircraft performance data are used to improve the accuracy of the ATM DST performance models for altitude profile (ascent and descent) predictions. There are several options for defining this information, including: 1) actual thrust and drag models; 2) selected performance data (e.g., ascent/descent rates as a function of speed profile) for model calibration; or 3 ) thrust and drag calibration factors based on a predefined baseline for a particular aircraft type. These user data would allow the ATM system to fine-tune its performance models on a per-flight/type basis.

Arrival Performance. Arrival performance data represent the arrival performance of a specific flight. Most important is the expected final approach speed, a key parameter for reducing excess spacing buffers between aircraft on final approach. ${ }^{6,17}$ Expected runway occupancy time, if known to the user, would also be useful for increasing airport capacity.

Pilot Procedures. Pilot procedure data are used to tune the ATM system's pilot procedure model to match the operation of a particular aircraft type or flight. Useful parameters include turn rates (or bank angles) as a function of aircraft state, and thrust management procedures (or target ascent/descent rates).

Atmospheric Characteristics. Atmospheric data include the predicted atmospheric state (wind and temperature) along the future route of flight as well as inflight measurements of atmospheric state. Updates of the predicted atmosphere are important for both user flight planning (ground and FMS) and ATM trajectory prediction. Wind and temperature profiles are needed for vertical-profile predictions, and the along-path wind component is critical for time-profile predictions. ${ }^{12}$ For extended terminal area applications, it may be useful for the ATM system to provide the atmospheric system updates. This approach would ensure precise agreement between user and ATM predictions, and use of the ATM system's knowledge of arrival-path constraints could improve the selection of the along-path wind profile for uplink to the FMS.

In-flight measurements of atmospheric state are useful for improvement of atmospheric modeling [e.g., the Rapid Update Cycle (RUC)] as well as near real-time updates of predictions based on the latest sensor measurements [e.g., Integrated Terminal Weather System (ITWS)]. ${ }^{18,19}$ Complementary data indicating aircraft maneuver state and wind components would be helpful for quality assurance of airborne wind measurements. The highest quality data are obtained when the aircraft is flying straight, and the along-track component is generally much more accurate than the cross-track component.

\section{$\underline{\text { ATM-System State Data }}$}

ATM-system state data are presented in Table 3, followed by a discussion of each item.

Table 3. ATM-System State Data

\begin{tabular}{ll}
\hline \hline \multicolumn{1}{c}{ Data } & Communication Direction \\
\hline Flight Constraints & User <- ATM System \\
Airspace State & User <- ATM System \\
Arrival Bank Status & User <- ATM System \\
Performance Analysis & User <-> ATM System \\
\hline \hline
\end{tabular}

Individual Flight Constraints. Individual flight constraints will enable users to determine viable preferences that do not conflict with known constraints (such as crossing restrictions). ${ }^{20}$ By providing this information to the users, the ATM system helps users to plan trajectories that are more consistent with the state of the ATM system.

Airspace State. Airspace state data describe the condition of the airspace of interest to a particular flight. Example characteristics include limited capacity (due to adverse weather and/or high aircraft density), estimated delay, and the status of special-use airspace. Airspacestate information will enable users to adapt flight plans to avoid predictable bottlenecks.

Bank Status. Bank status data describe the status of an arrival bank in terms of the sequence, ETA, and delay of each flight. The uniqueness of this information is that the data are based on the actual and planned actions of the ATM system. Users may use these data to improve the planning and management of arrival banks. Accurate knowledge of arrival bank status over the last 30 to 60 minutes of flight will enable a hub operation to optimize its use of ground-service resources. Individual flightdelay information will also assist users in deciding if and when a flight should be directed.

Flight/Airspace Performance Analysis. Flight/airspace performance analysis data represent the actual time histories of individual flight deviations and delay as functions of time and event (such as airborne delays and weather). Figure 3 presents an example time history that could be produced by ATM DSTs such as CTAS. This combination of user and ATM-system data [in addition to the out, off, on, in (OOOI) times for each 
flight] would provide the users and ATM system with valuable feedback that clearly identifies the factors that impacted user operations within a particular airspace. Analysis of these data over a period of time will provide users with insight into the precise conditions that determine the average time of flight, and variation, for a particular city pair. These data could be useful for minimizing buffer time planned into airline schedules as well as the determination of the factors leading to delay.

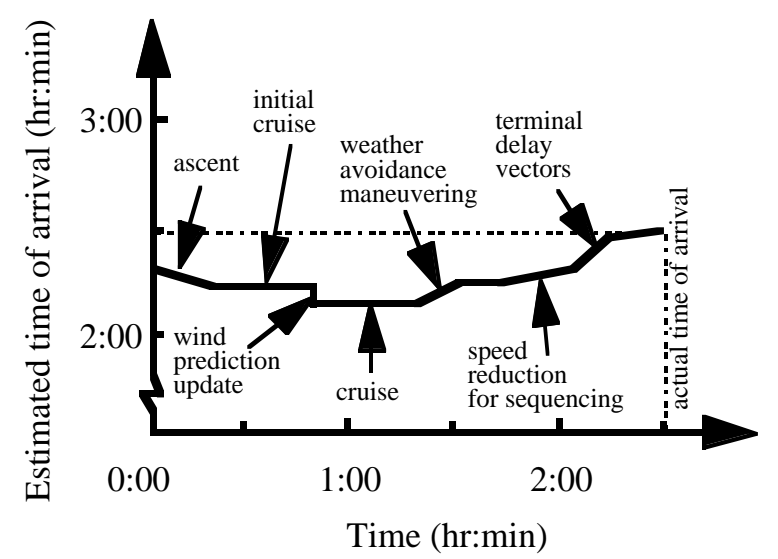

Figure 3. Example estimated time of arrival history.

\section{Experimental Evaluations}

The data exchange concepts described in this paper are being implemented for experimental evaluation by NASA. One set of tests, conducted under the NASA Terminal Area Productivity (TAP) Program, will investigate CTAS-FMS integration using the experimental FMS and data-link capability of the NASA Transport Research Facility (TRF) Boeing 757. A second set of tests will investigate the integration of CTAS with the FMS and Airline Operation Control (AOC) using currently available FMS and data-link technology.

\section{TAP Experiments}

The TAP experiments will investigate the procedures and system performance issues associated with 4D trajectory negotiation in en-route airspace and CTAS-based FMSroute clearances in the terminal area. A diagram of the basic scenario message sequence is given in figure 4 .

User-preference information is provided in the initial Flight-Plan and automatic User-Preference downlinks. Aircraft state (not shown in the figure) is continuously provided through Automatic Dependent Surveillance (ADS)-type downlinks. Additional user-supplied calibration data is provided in the Flight-Plan and UserPreference downlinks while ATM-supplied calibration data is provided in the CTAS-Planning uplink. ATM state information is provided by the CTAS Planning and CTAS
Constraints uplinks. The ATC clearances are delivered via the CTAS Clearance uplinks.

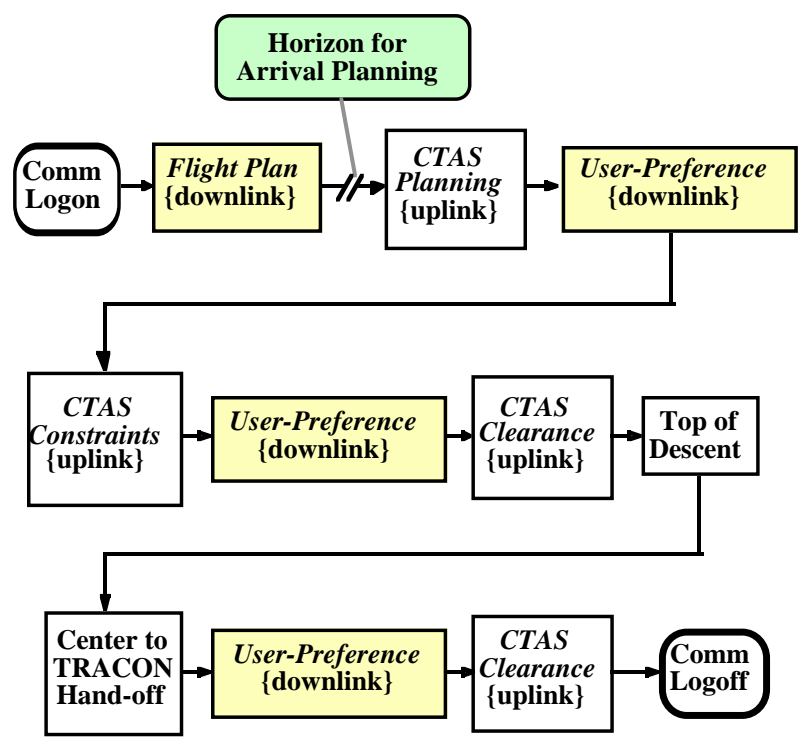

Figure 4. Message and event sequence of the TAP data exchange scenario.

Existing and proposed aeronautical data-link applications have been reviewed specifically to determine their suitability to support this data exchange. Modifications to existing Controller Pilot Data-Link Communication (CPDLC) and ADS message sets, to enable the TAP scenarios, have been identified. These modifications include both new messages and data types as well as changes to the initiation of ADS and CPDLC downlinks.

The initial TAP experiments are focusing on procedures and system performance associated with the CTAS clearance aspects of the data-exchange scenario. Future experiments will use this scenario to specifically address the exchange of user-preference, calibration, and ATMstate data.

\section{$\underline{\text { Operational Field Evaluation }}$}

To complement the TAP experimental system approach, a "near-operational" system is proposed to be assembled using available FMS and data-link technology. Figure 5 depicts the major components of the system to be assembled. Although the Aircraft Communications Addressing and Reporting System (ACARS) data link may not be suitable for operational CPDLC, it would provide an excellent platform for involving users in nearterm field testing. The recent generation of commercial FMS systems, which are integrated with ACARS communications, will allow for many data parameters to be exchanged with minimum pilot workload. 
The evaluation goals are to (1) integrate flight-deck, flight-planning [airline operational control (AOC)], and CTAS tools, and (2) explore promising CTAS dataexchange applications. The plan is to extend CTAS field trials to explore the benefits and impact of data exchange on CTAS computations and user flight operations. Live traffic testing will begin with "passive" CTAS applications (shadow analysis of CTAS advisories) with a transition to the issuance of CTAS-based clearance constraints for selected flights. The data will include user preferences such as speed profile or cost index. Usersupplied calibration data will include aircraft position, speed, weight, wind, temperature, and trajectory intent. CTAS will provide the user with updates of wind along the selected arrival path as well as waypoint constraints for top of descent or crossing restrictions. As in earlier CTAS field tests, these data-link evaluations would be conducted with minimum impact on the users and ATC facility.

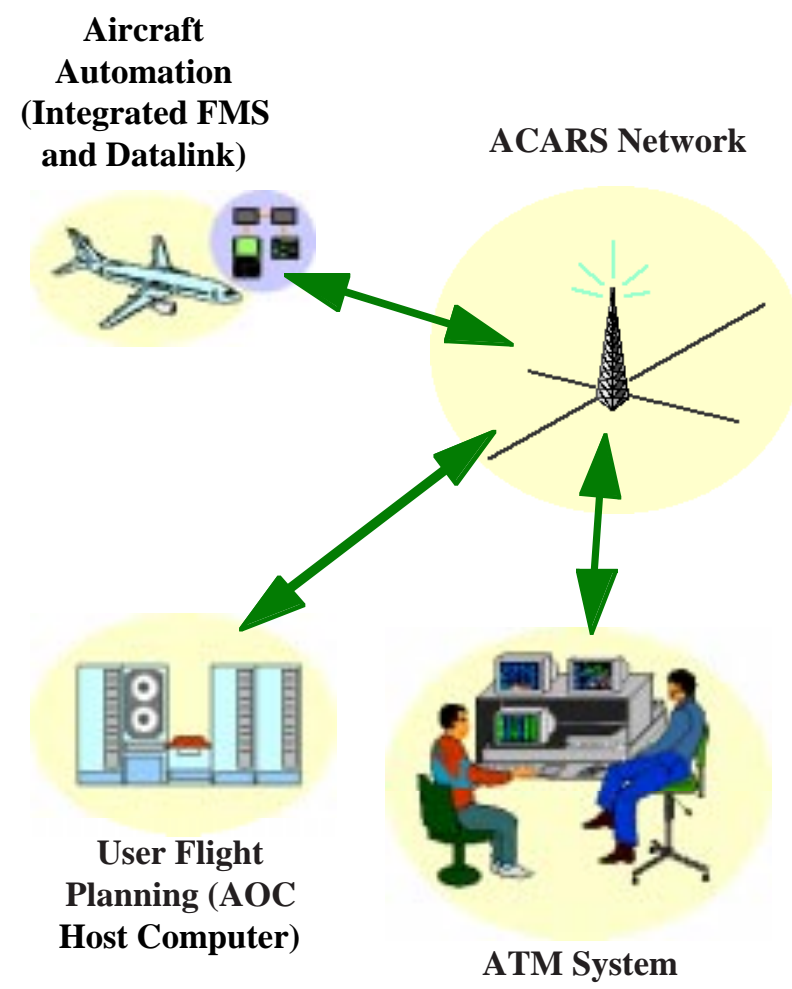

Figure 5. System components for user-ATM data exchange.

\section{Concluding Remarks}

The data-exchange concept has the potential to achieve free-flight benefits (improved operating efficiencies), as defined by the individual airspace users. Data exchange integrates user and ATM systems in three ways: by increasing the visibility of user preferences in the ATM system, independent of user capability; by providing users with ATM-state information to support the selection of viable preference options; and by providing all parties with the most accurate source of data for trajectory prediction. If ATM decision support tools are designed to accurately incorporate user preferences into their realtime traffic analysis, then the clearance advisories will be able to favor the user's preferences with minimum deviations due to conflicting preferences. The agency approach of incorporating preferences in the ATM tools will provide the foundation for ATM to offer userpreference service for users of varying capability while providing a mechanism for users to take advantage of higher levels of automation (such as flight-planning tools, FMS, and data link) if they so choose to equip. The two experiments described will provide valuable insight for integrating future ATM tools with current user systems (FMS, flight planning, and data link) as well as the design and integration of future ATM and user automation.

\section{$\underline{\text { Acknowledgments }}$}

The authors appreciate the assistance of Roger Beatty (American Airlines), Rusty Bell (Delta Airlines), and Ron Smith and Chris Pear (United Airlines) in sharing their insights into the concerns of airline operational control.

\section{$\underline{\text { References }}$}

1 RTCA, Inc.: "Free Flight Implementation," Final Report of RTCA Task Force 3, Washington D.C., October 26, 1995.

2 Vivona, R., Ballin, M., Green, S., Bach, R., and McNally, D.: "Overview of a Ground-Based System Concept for Supporting Increased User Preference," to be presented at the AIAA Conference on Guidance, Navigation, and Control, New Orleans, LA, August 1997.

3 Erzberger, H. and Nedell, W.: "Design of an Automated System of Arrival Traffic," NASA TM102201, June 1989.

4 Erzberger, H.: "Design Principles and Algorithms for Automated Air Traffic Management," AGARD Lecture Series No. 200 on Knowledge-based Functions in Aerospace Systems, Madrid, Paris, San Francisco, November 1995.

5 Green, S., den Braven, W., and Williams, D.: "Development and Evaluation of a Profile Negotiation Process for Integrating Aircraft and Air Traffic Control Automation," NASA TM-4360, April 1993.

6 Ballin, M., and Erzberger,H.: "An Analysis of Landing Rates and Separations at the Dallas /Fort Worth International Airport," NASA TM-110397, July 1996.

7 Hunter, G., Weidner, T., Couluris, G., Sorensen, J., and Bortins, R.: "CTAS Error Sensitivity, Fuel 
Efficiency, and Throughput Benefits Analysis," Seagull Technology, Inc. Report 96150-02, July 1996.

8 Swenson, H., Hoang, T., Engelland, S., Vincent, D., Sanders, T., Sanford, B., and Heere, K.: "Design and Operational Evaluation of the Traffic Management Advisor at the Ft. Worth Air Route Traffic Control Center," to be presented at the USA/Europe ATM Research and Development Seminar, Paris, France, June 1997.

9 Green, S.M., and Vivona, R.: "Field Evaluation of Descent Advisor Trajectory Prediction Accuracy," Proceedings of the AIAA Conference on Guidance, Navigation, and Control, San Diego, CA, July 29-31, 1996.

10 Erzberger, H.: "Design Principles and Algorithms for Automated Air Traffic Management," in AGARD Lecture Series No. 200, Knowledge-Based Functions in Aerospace Systems, Madrid, Paris, San Francisco November 1995.

11 Paielli, R., and Erzberger,H.: "Conflict Probability Estimation for Free Flight," NASA TM-110411, October 1996.

12 Williams, D.H., and Green, S.M.: "Flight Evaluation of the Center/TRACON Automation System Trajectory Prediction Process," in preparation as a NASA paper.

13 Wong, G.: “The Dynamic Planner," in preparation as a NASA paper.

14 RTCA, Inc.: "Minimum Operational Performance Standards for Airborne Automatic Dependent Surveillance (ADS) Equipment," Special Committee 170 DO-212, October 1992.

15 RTCA, Inc.: "Minimum Operational Performance Standards for ATC Two Way Data Link Communications," Special Committee 169 DO-219, August 1993.

16 RTCA, Inc.: "Minimum Aviation System Performance Standards for Air Traffic Management (ATM) Aeronautical Operational Control (AOC) - GroundGround Information Exchange," Special Committee 169 Working Group 5 Draft, March 1997.

17 Credeur, L., Capron, W.R., Lohr, G.W, Crawford, D.J., Tang, D.A., and Rodgers, W.G.: "FinalApproach Spacing Aids (FASA) Evaluation for Terminal-Area, Time-Based Air Traffic Control," NASA TP-3399, December 1993.

18 Benjamin, S. G., Brundage, K. J., and Morone, L. L.: "The Rapid Update Cycle. Part I: Analysis Model Description," Technical Procedures Bulletin No. 416, NOAA/NWS, 1994.
19 Cole, R.E., and Wilson, F.W.: "ITWS Gridded Winds Product," 6th Conference on Aviation Weather Systems, American Meteorological Society, 1995.

20 Palmer, E., Crane, B., Johnson, N., Smith, N., Feary, M., Cashion, P., Goka, T., Green, S., and Sanford, B.: "Field Evaluation of Flight Deck Procedures for Flying CTAS Descents," Presented at the Ninth International Symposium on Aviation Psychology, Columbus, Ohio, Apr. 1997. 